AIP $\mid \begin{aligned} & \text { Jumalot } \\ & \text { Applied Physics }\end{aligned}$

\title{
Supercurrent in $\mathrm{Nb} / \mathrm{InAs}$-nanowire/Nb Josephson junctions
}

H. Y. Günel, I. E. Batov, H. Hardtdegen, K. Sladek, A. Winden et al.

Citation: J. Appl. Phys. 112, 034316 (2012); doi: 10.1063/1.4745024

View online: http://dx.doi.org/10.1063/1.4745024

View Table of Contents: http://jap.aip.org/resource/1/JAPIAU/v112/i3

Published by the American Institute of Physics.

\section{Additional information on J. Appl. Phys.}

Journal Homepage: http://jap.aip.org/

Journal Information: http://jap.aip.org/about/about_the_journal

Top downloads: http://jap.aip.org/features/most_downloaded

Information for Authors: http://jap.aip.org/authors

\section{ADVERTISEMENT}

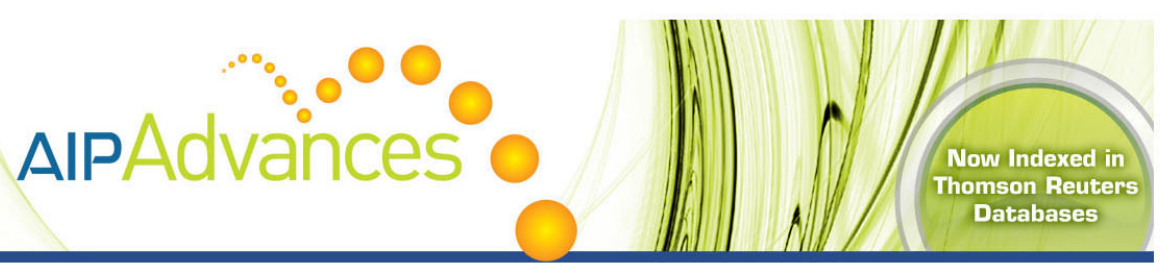

\section{Explore AIP's open access journal: Rapid publication \\ - Article-level metrics \\ - Post-publication rating and commenting}




\title{
Supercurrent in $\mathrm{Nb} / \mathrm{InAs}-n a n o w i r e / \mathrm{Nb}$ Josephson junctions
}

\author{
H. Y. Günel, ${ }^{1}$ I. E. Batov, ${ }^{2}$ H. Hardtdegen, ${ }^{1}$ K. Sladek, ${ }^{1}$ A. Winden, ${ }^{1}$ K. Weis, ${ }^{1}$ G. Panaitov, ${ }^{3}$ \\ D. Grützmacher, ${ }^{1}$ and Th. Schäpers ${ }^{1,4}$ \\ ${ }^{1}$ Peter Grünberg Institute (PGI-9) and JARA-Fundamentals of Future Information Technology, \\ Forschungszentrum Jülich GmbH, 52425 Jülich, Germany \\ ${ }^{2}$ Institute of Solid State Physics, Russian Academy of Sciences, Chernogolovka, 142432 Moscow, Russia \\ ${ }^{3}$ Peter Grünberg Institute (PGI-9) and JARA-Fundamentals of Future Information Technology, \\ Forschungszentrum Jülich GmbH, 52425 Jülich, Germany \\ ${ }^{4}$ II. Physikalisches Institut, RWTH Aachen University, 52056 Aachen, Germany
}

(Received 8 May 2012; accepted 11 July 2012; published online 10 August 2012)

\begin{abstract}
We report on the fabrication and measurements of planar mesoscopic Josephson junctions formed by InAs nanowires coupled to superconducting $\mathrm{Nb}$ terminals. The use of Si-doped InAs-nanowires with different bulk carrier concentrations allowed to tune the properties of the junctions. We have studied the junction characteristics as a function of temperature, gate voltage, and magnetic field. For junctions with high doping concentrations in the nanowire, Josephson supercurrent values up to $100 \mathrm{nA}$ are found. Owing to the use of $\mathrm{Nb}$ as superconductor, the Josephson coupling persists at temperatures up to $4 \mathrm{~K}$. In all junctions, the critical current monotonously decreased with the magnetic field, which can be explained by a recently developed theoretical model for the proximity effect in ultra-small Josephson junctions. For the low-doped Josephson junctions, a control of the critical current by varying the gate voltage has been demonstrated. We have studied conductance fluctuations in nanowires coupled to superconducting and normal metal terminals. The conductance fluctuation amplitude is found to be about 6 times larger in superconducting contacted nanowires. The enhancement of the conductance fluctuations is attributed to phasecoherent Andreev reflection as well as to the large number of phase-coherent channels due to the large superconducting gap of the Nb electrodes. (C) 2012 American Institute of Physics.

[http://dx.doi.org/10.1063/1.4745024]
\end{abstract}

\section{INTRODUCTION}

Coherent transport in mesoscopic semiconductor-based Josephson junctions has been attracting a lot of interest from fundamental and applied physics point of view. Modern nanofabrication techniques provide a possibility to fabricate planar multi-terminal Josephson structures relevant for the realization of different types of hybrid superconductor/ semiconductor nanoscale devices. ${ }^{1,2}$ Josephson field effect transistors, ${ }^{3}$ superconducting quantum point contacts, ${ }^{4}$ and injection current controlled Josephson junctions ${ }^{5}$ were realized with high-mobility 2-dimensional electron gases in semiconductor heterostructures. Recently, the Josephson effect was also observed in nanoscale devices formed by semiconductor nanowires coupled to superconducting terminals. ${ }^{1,6-11}$ Very recently, semiconductor nanowires had also been used for experiments dedicated to confirm the existence of Majorana fermions. ${ }^{12-14}$

Up to now, most of experimental studies of semiconductor-based Josephson junctions at nanoscale have been focused on structures with an InAs-nanowire as a semiconductor weak link. Experiments show that a charge accumulation layer is formed at the surface of the InAs, ${ }^{15}$ which provides a sufficiently low resistive contact to superconducting electrodes. It was found that highly transparent contacts are formed at the interfaces between the InAs nanowire and Al superconducting electrodes. At temperatures below $1 \mathrm{~K}$, the high transparency of the contacts gives rise to proximityinduced superconductivity. A large number of experiments have been carried out on Al/InAs-nanowire junctions, demonstrating tunable Josephson supercurrents, ${ }^{6,7}$ supercurrent reversal, ${ }^{8}$ Kondo enhanced Andreev tunneling, ${ }^{16}$ and suppression of supercurrent by hot-electron injection. ${ }^{10}$ Furthermore, gate-controlled superconducting quantum interference devices (SQUIDs) (Refs. 1 and 8) and tunable Cooper pair splitters ${ }^{17}$ have been realized.

The use of $\mathrm{Al}$ as a superconductor in nanowire-based Josephson junctions limits the operation temperature of the nanofabricated devices below $T \approx 1.2 \mathrm{~K}$. To extend the operation of the Josephson devices at higher temperatures, it has been suggested to use superconductors which have higher temperatures of superconducting transition. Recently, Spatnis et al. ${ }^{18}$ have realized proximity DC SQUIDs based on InAs nanowires and vanadium superconducting electrodes $\left(T_{c} \approx 4.6 \mathrm{~K}\right)$. It is shown that V/InAs-nanowire/V Josephson junctions can operate at temperatures up to $2.5 \mathrm{~K}$. Furthermore, for InN-nanowire-based junctions with $\mathrm{Nb}$ electrodes ( $T_{c} \approx 9.3 \mathrm{~K}$ ), a Josephson supercurrent was observed up to temperatures of $3.5 \mathrm{~K}$. $^{9}$

In some aspects, small-size planar superconductor/ normal conductor/superconductor (SNS) Josephson junctions differ significantly from their large-size counterparts, i.e., the magnetic field dependence of the critical current $I_{c}$ is expected to decrease monotonously with increasing magnetic field $B$ in contrast to the Fraunhofer-type $I_{c}$ vs. $B$ dependence for larger junctions. ${ }^{19,20}$ Indeed, a monotonous decrease of $I_{C}$ was recently observed in $\mathrm{Nb} / \mathrm{Au} / \mathrm{Nb}$ and $\mathrm{Al} / \mathrm{Au} / \mathrm{Al}$ junctions 

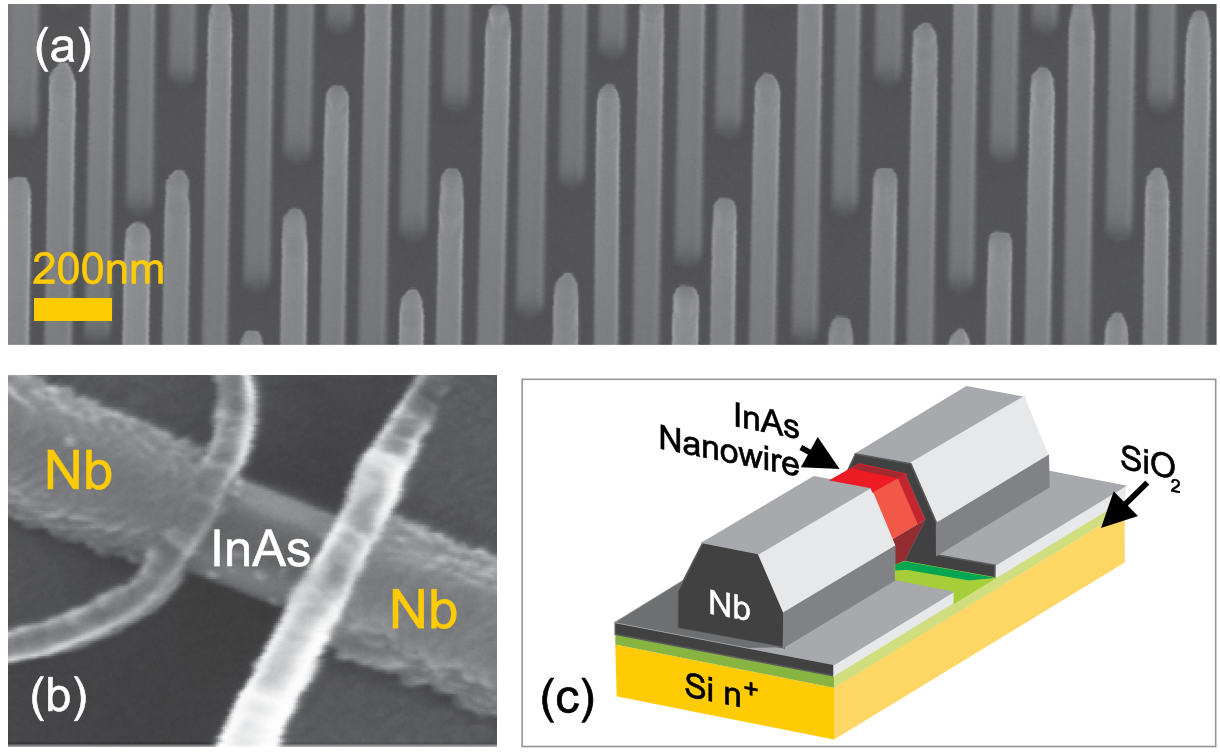

FIG. 1. (a) Scanning electron micrograph of the as grown nanowires with doping factor 500. (b) Scanning electron micrograph of sample L2. (c) Schematic illustration of the junction layout. as well as in $\mathrm{Nb} / \mathrm{InN}$-nanowire/Nb junctions. ${ }^{9,21}$ Furthermore, in junctions with a semiconductor nanowire in between two superconducting electrodes mesoscopic effects, i.e., conductance fluctuations and fluctuations of the critical current have been observed when the gate voltage is varied. ${ }^{6,22,23}$ The gate voltage dependent conductance fluctuations measured at finite bias voltage were shown to follow almost precisely the fluctuations of the supercurrent. ${ }^{6,24}$ It was also found that the conductance fluctuation amplitude measured at bias voltages lower than $2 \Delta / \mathrm{e}$ significantly exceeds the amplitude of the normal-state universal conductance fluctuations. ${ }^{22,23}$

In our study, we investigated the Josephson effect in $\mathrm{Nb} / \mathrm{InAs}$-nanowire/Nb hybrid structures. By using InAsnanowires with two different bulk carrier concentrations, we were able to vary the properties of the nanoscale weak link Josephson junction to a large extent, i.e., in the junctions with the highly doped InAs nanowires a relatively large Josephson supercurrent is observed, whereas for junctions with low doped nanowires $I_{C}$ is reduced but here a gate control of the superconducting switching currents is possible. We devoted special attention to effects which originate from the small size of the junctions, in particular, the magnetic field dependence of the critical current as well as the gate voltage dependent conductance and supercurrent fluctuations. The field dependence of the critical current and the fluctuation phenomena are compared to recent theoretical models.

\section{EXPERIMENTAL}

The $n$-type doped InAs nanowires were grown by selective area metal organic vapor phase epitaxy (MOVPE) without using catalyst material. In order to tune the Si doping level, the ratio of disilane $\left(\mathrm{Si}_{2} \mathrm{H}_{6}\right)$ partial pressure and group III precursor has been adjusted as $p\left(\mathrm{Si}_{2} \mathrm{H}_{6}\right) / p$ (TMIn) $=7.5 \times 10^{-5}$, which is defined as doping factor 1 . In this study, we have used nanowires with two different doping concentrations. The resistivity for the lower (doping factor 100 , carrier concentration $n_{3 d} \approx 1 \times 10^{18} \mathrm{~cm}^{-3}$ ) and higher doped (doping factor $500, n_{3 d} \approx 7 \times 10^{18} \mathrm{~cm}^{-3}$ ) nanowires was 0.019 and $0.0018 \Omega \mathrm{cm}$, respectively. The field-effect mobility of the nanowire with the lower doping concentration is about $970 \mathrm{~cm}^{2} / \mathrm{Vs}$. Owing to insufficient gate control, no mobility value could be determined for the wire with higher doping concentration. In Fig. 1(a), a scanning electron micrograph of the as-grown nanowires is shown. Detailed information concerning the growth, characterization, and transport parameters of the nanowires can be found elsewhere. ${ }^{25,26}$ The samples are labeled as "L1, L2" and "H1, H2" for junctions based on a nanowire with lower (doping factor 100) or higher (doping factor 500) doping concentration, respectively.

In order to contact the nanowires with $\mathrm{Nb}$ electrodes, they were transferred from the growth substrate to an $n^{+}-\mathrm{Si} / \mathrm{SiO}_{2}$ substrate with predefined electron beam markers. The $\mathrm{Nb}$ electrodes were defined by electron beam lithography and lift-off. Before $\mathrm{Nb}$ deposition, the samples were exposed to an oxygen plasma to remove electron beam resist residues on the contact area. Furthermore, in order to obtain a high contact transparency, $\mathrm{Ar}^{+}$ion milling was employed to remove the native oxide on the nanowire surface. The $100 \mathrm{~nm}$ thick $\mathrm{Nb}$ layer was deposited by sputtering. The highly doped $n$-type substrate was used for back-gating of the nanowires. A scanning electron micrograph of a typical junction (sample L2) is shown in Fig. 1(b), while a schematics of the junction layout can be found in Fig. 1(c). The contact separations $L$ of the four different samples are given in Table I. The transport measurements were performed at temperatures down to $0.3 \mathrm{~K}$ in a He-3 cryostat equipped with a superconducting solenoid with a magnetic field up to $7 \mathrm{~T}$. The DC and differential current-voltage characteristics were measured using a four-terminal current-driven measurement scheme. Current and voltage leads were filtered by RC filters thermally anchored at $\sim 2 \mathrm{~K}$. The differential resistance $d V / d I$ was measured with a lock-in amplifier by superimposing a small $\mathrm{AC}$ signal of $5 \mathrm{nA}$ to the junction bias current.

\section{RESULTS AND DISCUSSION}

In Fig. 2(a), the current-voltage (IV) characteristics at various temperatures between 0.4 and $4.8 \mathrm{~K}$ are shown for a 
TABLE I. Sample parameters: The contact separation, $L$, and the diameter of nanowires, $d$, have been determined from scanning electron micrograph; $I_{C}$ is the critical current and $R_{N}$ is the normal state resistance of the samples.

\begin{tabular}{lcccc}
\hline \hline Device & $L(\mathrm{~nm})$ & $d(\mathrm{~nm})$ & $R_{N}(\mathrm{k} \Omega)$ & $I_{c}(\mathrm{nA})$ \\
\hline Sample H1 & 140 & 110 & 0.75 & 100 \\
Sample H2 & 160 & 100 & 0.90 & 70 \\
Sample L1 & 70 & 80 & 3.8 & 2.8 \\
Sample L2 & 85 & 75 & 2.5 & 12 \\
\hline \hline
\end{tabular}

sample with a highly doped nanowire (sample H1). At temperatures $T \leq 4 \mathrm{~K}$ and small bias, a clear Josephson supercurrent is observed in the junction. As the bias current exceeds a certain value $I_{s w}$, the Josephson junction switches from the superconducting to the normal state. The switching current $I_{s w}$ measured at $0.4 \mathrm{~K}$ for the sample $\mathrm{H} 1$ is about $100 \mathrm{nA}$. With increasing the temperature $I_{s w}$ is reduced. At $T>4.5 \mathrm{~K}$, the supercurrent is suppressed completely. Our measurements show that at temperatures below $2 \mathrm{~K}$ the $I V$-characteristics are hysteretic. With increasing the temperature the hysteresis is gradually suppressed. The retrapping current $I_{r}$, defined by the switching from the normal state back into the superconducting state, has a value of $84 \mathrm{nA}$ at $0.4 \mathrm{~K}$ and remains almost constant when the temperature is increased to $2 \mathrm{~K}$. As can be inferred from the $I V$-characteristics
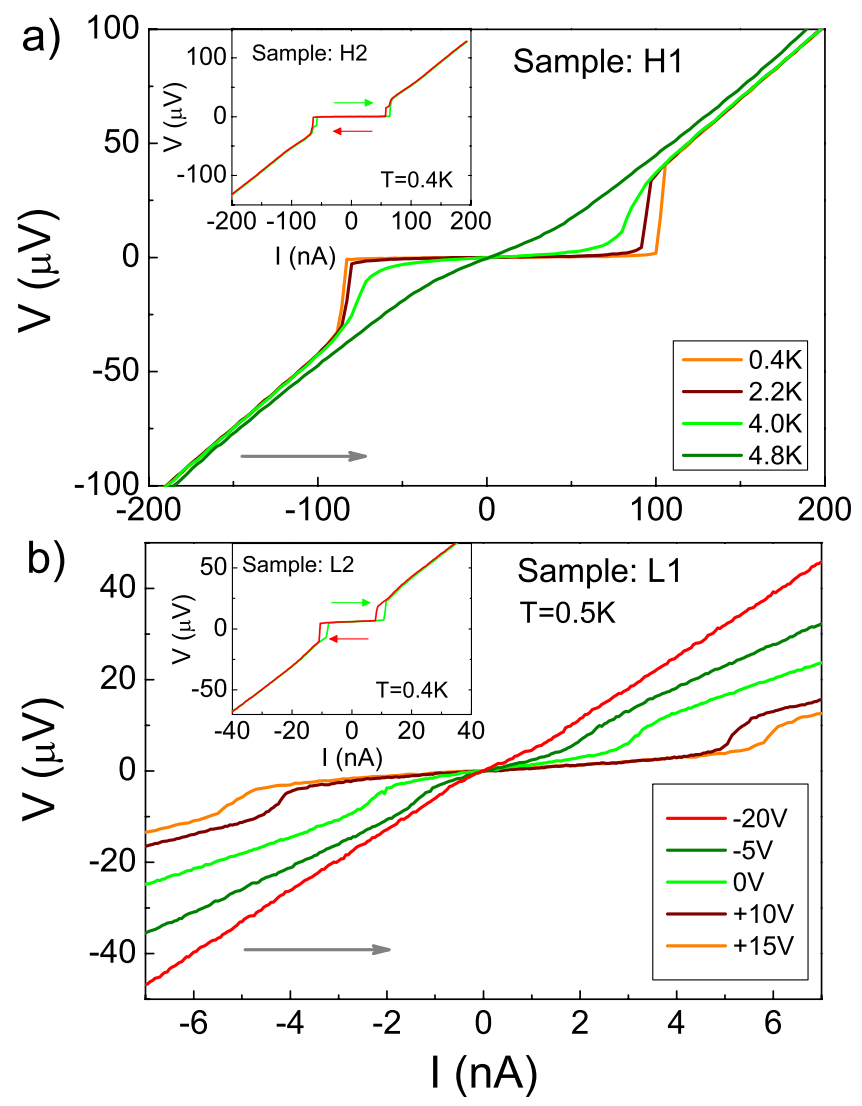

FIG. 2. (a) $I V$-characteristics of a highly doped device (sample H1) at various temperatures. The inset shows the $I V$-characteristics of the second highly doped device (sample $\mathrm{H} 2$ ) at $0.4 \mathrm{~K}$. (b) Gate voltage dependent $I V$-characteristics of the junction with the low doped nanowire (sample L1) at $0.5 \mathrm{~K}$, the inset shows the $I V$-characteristics of the second device with a low-doped nanowire (sample L2) at zero gate voltage. The arrows in (a) and (b) indicate the sweep direction. shown in Fig. 2(a) (inset), the switching current $I_{s w}$ of the second sample with the highly doped nanowire (sample H2) has a somewhat smaller value of $70 \mathrm{nA}$ compared to the first sample.

It should be noted that the switching current $I_{S w}$ is strongly affected by the external on-chip $R C$ circuit integrated with the Josephson junction ${ }^{23,27,28}$ and depends on the quality factor $Q$ of the devices. Following the approach given in Refs. 23 and 27, we have estimated the quality factor $Q$ of the samples $\mathrm{H} 1, \mathrm{H} 2$ taking into account the electromagnetic environment. Estimations show that $Q<1$, thus, the junctions $\mathrm{H} 1, \mathrm{H} 2$ are in the overdamped limit and the measurable supercurrent $I_{s w}$ approaches the thermodynamic critical current $I_{c}$. The observed hysteresis in the $I V$ characteristics in our planar mesoscopic SNS Josephson junctions can be explained by the increase of the electron temperature in the weak link once the junction switches to the resistive state. $^{29}$

Due to the lower electron concentration, gate control was achieved for the samples with the lower doped nanowires. For sample L1, this is shown in Fig. 2(b), where the $I V$-characteristics at $0.5 \mathrm{~K}$ are plotted for back-gate voltages between $-20 \mathrm{~V}$ and $+15 \mathrm{~V}$. At zero gate voltage, the measured $I_{c}$ is about $2.8 \mathrm{nA}$, which is considerably smaller than the $I_{c}$ of the junctions with the highly doped nanowires. We attribute the lower $I_{c}$ to the larger resistivity of the lower doped nanowires. As can be seen in Fig. 2(b), by applying a back-gate voltage of $+15 \mathrm{~V}$ the switching current can be increased by about a factor of 2 . The larger value of the switching current is due to the fact that by applying a positive gate voltage the electron concentration in the nanowires is increased so that the resistivity is decreased. In contrast, by applying a negative gate voltage the electron concentration in the nanowire is reduced. As a consequence, $I_{c}$ is reduced [cf. Fig. 2(b)], and at a gate voltage of $-20 \mathrm{~V}$, the supercurrent is suppressed completely. As can be seen in Fig. 2(b), a small voltage drop appears for currents below $I_{c}$ which can be attribute thermal smearing. A Josephson supercurrent was also observed for the second sample with a low doped nanowire (sample L2) [cf. Fig. 2(b) (inset)]. Here, $I_{c}$ is found to be $12 \mathrm{nA}$ at $0.4 \mathrm{~K}$ and thus larger than the value for sample L1. As discussed in detail below, for sample L2 a gate control of $I_{c}$ was obtained as well.

In Fig. 3, the differential resistance $d V / d I$ vs bias voltage of the sample L2 is shown. The measurement temperature was $0.3 \mathrm{~K}$. The peaks in $d V / d I$ at finite bias voltages can be associated with the subharmonic energy gap structure caused by multiple Andreev reflections, with peak positions given by $V_{n}=2 \Delta /$ en $(n=1,2, \ldots) \cdot{ }^{30-32}$ From the fit of the peak positions, we have determined the superconducting energy gap $\Delta$ in the $\mathrm{Nb}$ leads $\Delta=1.2 \mathrm{meV}$ and found that the observed peaks correspond to $n=1,2$, and 4 . The contact transparency in the device L2 can be estimated using the $I V$-characteristic for $V>2 \Delta / e^{7,11}$ We have found that the fit to the $I V$-curve at the normal state in the range $V>2.5 \mathrm{mV}$ extrapolates to a finite excess current $I_{\text {exc }}=195 \mathrm{nA}$ as shown in the inset of Fig. 3. Using the superconducting energy gap $\Delta=1.2 \mathrm{meV}$ and the normal state resistance of the junction $R_{n}=2.47 \mathrm{k} \Omega$, we obtain $e I_{\text {exc }} R_{n} / \Delta=0.4$. We analyzed the 


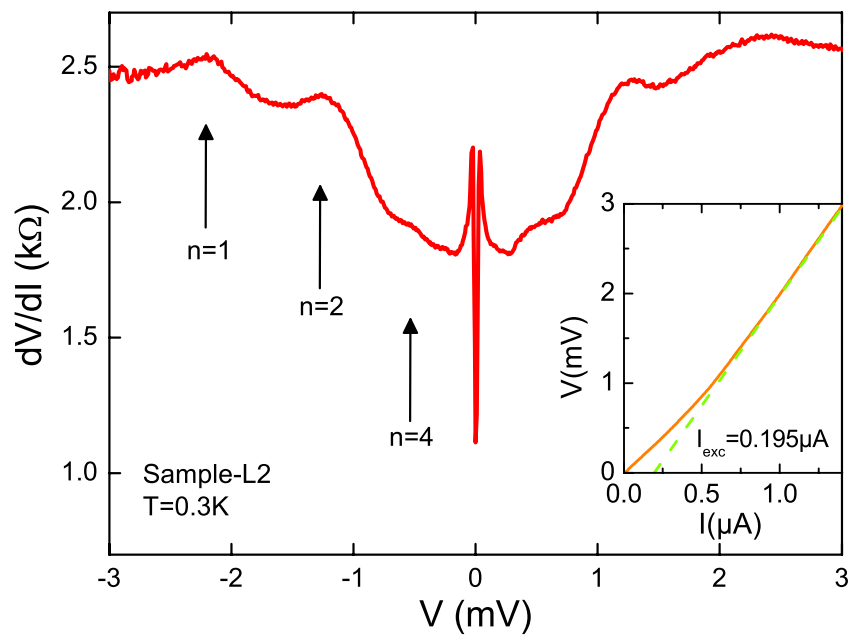

FIG. 3. Differential resistance $d V / d I$ versus bias voltage for sample L2 at $0.3 \mathrm{~K}$. The subgap features are indicated by arrows. The inset shows a high bias range $I V$-characteristic of the device (solid line) and the linear fit of the $I V$-curve at $V>2 \Delta$ (dashed line) to show the excess current value $I_{\text {exc }}$.

data within the framework of the standard Blonder-TinkhamKlapwijk (BTK) theory. ${ }^{33}$ The obtained value $e I_{\text {exc }} R_{n} / \Delta=$ 0.4 is converted to the BTK barrier strength parameter $Z \approx 0.85,{ }^{31}$ which corresponds to a contact transparency $T_{n} \approx 0.6$. Subgap features as found here have been observed before in other superconductor-semiconductor junctions. 9,34,35 From the electron-phonon coupling strength $^{9,36}$ $2 \Delta_{0} / \mathrm{k}_{B} \mathrm{~T}_{c}=3.9$ and assuming $\Delta_{0}=1.2 \mathrm{meV}$ for the superconducting gap at $T=0$, we obtain a critical temperature $T_{c}=7.2 \mathrm{~K}$ for the $\mathrm{Nb}$ electrodes, which fits well to the measured value of $T_{c}$.

Owing to the large critical field of $\mathrm{Nb}$, a Josephson supercurrent is maintained up to relatively large magnetic fields. This is illustrated in Fig. 4 (inset), where the colorscaled voltage drop measured for sample $\mathrm{H} 2$ is given as a function of magnetic field and bias current. The measurement temperature was $0.4 \mathrm{~K}$. The magnetic field was applied perpendicular to the substrate. The resulting values of $I_{c}$ normalized to the zero-field critical current $I_{c 0}$ are plotted as a

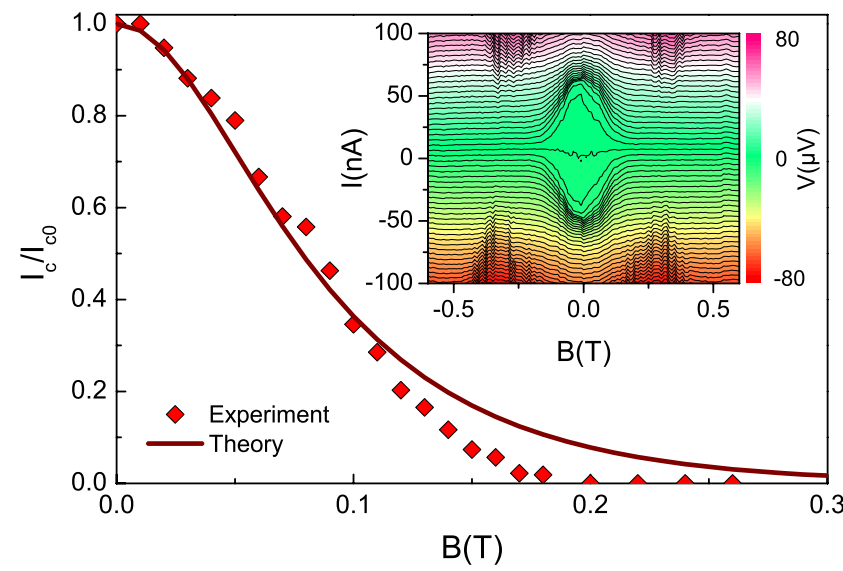

FIG. 4. Normalized critical current $I_{c} / I_{c 0}$ versus magnetic field $B(\diamond)$ for sample H2. The solid line represents the calculation according to the theoretical model. ${ }^{19,20}$ The inset shows the color-scaled voltage drop as a function of magnetic field and bias current. The contour lines correspond to constant voltage values separated by $2.5 \mu \mathrm{V}$. function of $B$ in Fig. 4. A monotonous decrease of the measured critical current $I_{c}$ with magnetic field $B$ is found. A complete suppression of $I_{c}$ occurred at about $0.2 \mathrm{~T}$. Earlier, a similar behavior of the critical current with magnetic field was observed in planar $\mathrm{Nb} / \mathrm{Au} / \mathrm{Nb}$ and $\mathrm{Al} / \mathrm{Au} / \mathrm{Al}$ Josephson junctions $^{21}$ and in InN nanowire-based Josephson junctions. ${ }^{9}$ The monotonous decrease of $I_{c}$ with increasing $B$ can be explained within the framework of a recently developed theoretical model for the proximity effect in diffusive narrowwidth Josephson junctions. ${ }^{19,20}$ There, it is shown that for junctions with a width comparable to or smaller than the magnetic length $\xi_{B}=\sqrt{\Phi_{0} / B}$, with $\Phi_{0}$ the magnetic flux quantum, the magnetic field acts as a pair-breaking factor that suppresses monotonously the proximity-induced superconductivity in the wire and the critical current.

For sample $\mathrm{H} 2$, the characteristic magnetic field $B_{0}$ defined by the flux quantum through the cross section of the normal wire, $B_{0}=\Phi_{0} / L d$, is as large as $0.13 \mathrm{~T}$, resulting in $\xi_{B}=126 \mathrm{~nm}$. The value of $\xi_{B}$ is thus comparable to the junction width, so that the theoretical model for the limit of narrow-width Josephson junctions can be applied. ${ }^{19,20} \mathrm{We}$ calculated the expected dependence of $I_{c}$ on $B$ using the Thouless energy defined by $E_{T h}=\hbar D / L^{2}$ as a fitting parameter. ${ }^{19,20,37}$ Here, $D$ is the diffusion constant and $L$ is the length of the junction. As can be seen in Fig. 4, a good agreement between experiment and theory is obtained. The best fit has been achieved for $E_{T h}^{f i t}=0.2 \mathrm{meV}$. This value is smaller than $E_{T h}=0.47 \mathrm{meV}$, determined from transport measurements in the normal state. We interpret the lower value of $E_{T h}^{f i t}$ as compared to the Thouless energy obtained from the transport data by the presence of interface barriers in the junctions.

In Fig. 5(a), the differential resistance $d V / d I$ of sample L2 is plotted in color scale as a function of bias current and back-gate voltage. The measurements were taken at $0.4 \mathrm{~K}$. It can be seen that on average the supercurrent range (black region) is reduced if a more negative gate voltage is applied. This is due to the corresponding decrease of the electron concentration in the nanowire. Since the current was biased from negative to positive values, the switching is nonsymmetric with respect to zero current, with the transition at negative and positive bias currents corresponding to the return current $I_{r}$ and the critical current $I_{c}$, respectively. A closer look on Fig. 5(a) reveals that $I_{c}$ fluctuates as a function of back-gate voltage. The corresponding values of $I_{c}$ are plotted in Fig. 5(b). The average amplitude of the critical current fluctuations, i.e., the root-mean-square (rms) of fluctuations over the applied gate voltage range, is found to be $\operatorname{rms}\left(\delta I_{c}\right) \approx 0.9 \mathrm{nA}$. The critical current fluctuations $\delta I_{c}$ were calculated by subtracting a linear increasing background current.

Mesoscopic fluctuations of the critical current have been theoretically studied mainly in two different regimes. For the short junction limit, it is found that the fluctuations are universal and that the fluctuation amplitude depends only on the superconducting gap, $\Delta: \delta I_{c} \sim e \Delta / \hbar .^{38-40}$ The limit of long Josephson junctions, where the Thouless energy $E_{T h}$ is much smaller than the superconducting gap in the leads has been investigated by Alt'shuler and Spivak. ${ }^{41}$ In this model, the 
(a)

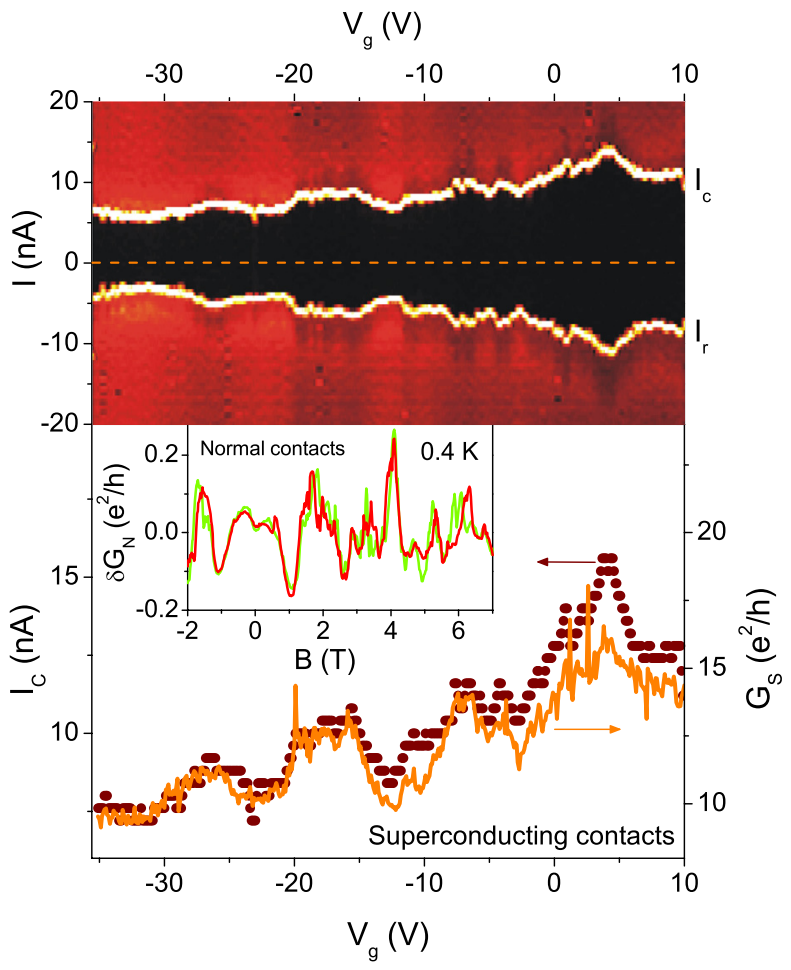

FIG. 5. (a) Differential resistance $(d V / d I)$ plotted as a function of bias current and gate voltage. (b) Fluctuations of the conductance $G_{S}$ and the critical current $I_{c}$ as a function of gate voltage. The inset graph shows two subsequent measurements of a normal contacted nanowire.

energy scale for mesoscopic fluctuations in the critical current, $I_{c}$, is set by the Thouless energy. Since in our case $E_{T h} \ll \Delta$, we compared the experimental values of $\operatorname{rms}\left(\delta I_{c}\right)$ with the model of Alt'shuler and Spivak which is appropriate for this particular regime. ${ }^{41}$ Within this model, the amplitude of supercurrent fluctuations at $T=0$ is given by $\operatorname{rms}\left(\delta I_{c}\right)$ $=0.60 e E_{T h} / \hbar$. By taking a Thouless energy $0.05 \mathrm{meV}$, as was estimated from the magnetic field dependence of $I_{c}$ for the sample L2, we obtain an expected fluctuation amplitude of $\operatorname{rms}\left(\delta I_{c}\right) \approx 7.3 \mathrm{nA}$. In the more recent model of Houzet and Skvortsov, ${ }^{42}$ the proximity effect and the resulting formation of a minigap in the normal conductor is included in the analysis of the critical current fluctuations. For the long junction limit, they obtained $\operatorname{rms}\left(\delta I_{c}\right)=1.49 E_{T h} e / \hbar$. According to this model, we find an even higher expected fluctuation amplitude of approximately $18 \mathrm{nA}$. For both models, the expected values of $\operatorname{rms}\left(\delta I_{c}\right)$ are considerably larger than the corresponding experimentally obtained values. The most probable reason of the lower measured value of $\operatorname{rms}\left(I_{c}\right)$ is the presence of a non-ideal superconductor/normal conductor interface, ${ }^{6,24}$ i.e., an interface barrier or different Fermi velocities in both materials. ${ }^{43}$ Both contributions lead to a decrease of $I_{c}$ and can be expected to result in an according decrease of $\operatorname{rms}\left(I_{c}\right)$.

In Fig. 5(b), the normalized differential conductance $G_{S}$ is plotted in units of $e^{2} / h$ as a function of back-gate voltage. The conductance values have been taken at a bias voltage $0.1 \mathrm{mV}$, which is well below $2 \Delta / e$, so that multiple Andreev reflections partially contribute to the total conductance. However, we did not attempt to drive the junction into a higher bias state above $2 \Delta / e$ or measure the fluctuations at temperatures above $T_{c}$, such that any superconducting properties are suppressed. The reason is that under these conditions the quasi-equilibrium phase coherent transport regime is left. As can be seen in Fig. 5(b), the fluctuation pattern of $G_{S}$ follows almost perfectly the pattern of the previously discussed fluctuations in $I_{c}$. A similar agreement between the fluctuation patterns of $I_{C}$ and $G_{S}$ has been observed before on $\mathrm{Al} / \mathrm{InAs}$-nanowire and on $\mathrm{Nb} / 2$-dimensional electron gas Josephson junctions. ${ }^{6,24}$

The fluctuations in $G_{S}$ originate from the phase-coherent transport through a conductor with small dimensions where only a limited number of scattering centers are involved. ${ }^{44-46}$ For our junction, we find a fluctuation amplitude of $\operatorname{rms}\left(\delta G_{S}\right)=1.18 e^{2} / h$, with $\delta G_{S}$ calculated by subtracting the linearly increasing background conductance. In order to compare $\operatorname{rms}\left(\delta G_{S}\right)$ obtained for a sample with superconducting electrodes with the corresponding value of $\operatorname{rms}\left(\delta G_{N}\right)$ of a normal conducting reference sample, we have contacted a nanowire from the same growth run with normal $\mathrm{Au} / \mathrm{Ti}$ electrodes. Here, the contact separation was $130 \mathrm{~nm}$. As shown in Fig. 5(b) (inset), at a temperature of $0.4 \mathrm{~K}$ reproducible conductance fluctuations are measured as a function of magnetic field. However, the average conductance fluctuation amplitude of $\operatorname{rms}\left(G_{N}\right)=0.2 e^{2} / h$ is significantly lower than the value found for the sample with superconducting electrodes. We attribute the enhanced conductance fluctuation observed for the sample with superconducting electrodes to the additional contribution of phase-coherent Andreev reflection. A similar behavior has been found in other proximity Josephson junctions as well. ${ }^{6,22,23,47,48}$ In Al-based Josephson junctions, an enhancement of the average fluctuation amplitude between 1.4 and 1.6 has been reported, ${ }^{47}$ whereas in our $\mathrm{Nb}$ contacted junctions, we found an enhancement by a factor of about 6 . The larger enhancement compared to the values reported for Al-based junctions can be qualitatively explained by the larger superconducting energy gap of $\mathrm{Nb}$ compared to $\mathrm{Al}$, resulting in a larger number of phasecoherent Andreev channels.

Finally, we would like to address the possibility of Majorana fermions that can be created by using a structure based on a superconducting electrode and a semiconductor nanowire. ${ }^{49,50}$ In fact, nanowires based on InAs comprise the necessary strong spin-orbit coupling ${ }^{25}$ and a large $g$-factor. ${ }^{51}$ Furthermore, by using $\mathrm{Nb}$ as a superconducting electrode a sufficiently large gap can be induced in the nanowire. ${ }^{12}$ Majorana fermions can be observed only at moderate magnetic fields, therefore, superconducting $\mathrm{Nb}$ electrodes offer an advantage due to its high critical magnetic field. Thus, InAs nanowires combined with $\mathrm{Nb}$ electrodes can be regarded as a suitable system for the creation of Majorana fermions.

\section{SUMMARY AND CONCLUSION}

In summary, we have successfully fabricated and characterized $\mathrm{Nb} / \mathrm{InAs}$-nanowire/Nb Josephson junctions. By taking advantage of $\mathrm{Nb}$ as a superconductor, we could demonstrate that the junctions comprising a highly doped InAs nanowire show a clear Josephson supercurrent up to 
relatively high temperatures of $4 \mathrm{~K}$. For the junctions with the lower doped nanowire, gate control of the Josephson supercurrent was achieved. The measurements of $I_{c}$ as a function of magnetic field show that a Josephson supercurrent can be maintained up to a field of $0.2 \mathrm{~T}$. The observed monotonous decrease of $I_{c}$ with increasing magnetic field is explained by the magnetic pair breaking effect in narrowwidth Josephson junctions. ${ }^{19,20}$ In the junctions with the lower doped nanowires, the Josephson supercurrent $I_{c}$ as well as the conductance $G_{S}$ fluctuates when the gate voltage is varied. The measured average amplitude of supercurrent fluctuations was smaller than the theoretically expected value. The large difference between both values is attributed to the presence of a barrier at the $\mathrm{Nb} /$ nanowire interface. The average conductance fluctuation amplitude for the $\mathrm{Nb} / \mathrm{InAs}$ nanowire samples was considerably larger than the corresponding value for a reference sample with normal conducting $\mathrm{Au} / \mathrm{Ti}$ contacts. We attribute this enhancement to the contribution of phase-coherent Andreev reflection.

\section{ACKNOWLEDGMENTS}

The authors are grateful to H. Kertz for assistance during the measurements and S. Trellenkamp for electron beam writing. H.Y.G. thanks The Scientific and Technological Research Council of Turkey (TUBITAK) foundation. I.E.B. acknowledges the Russian Foundation for Basic Research, Project No. RFBR 09-02-01499 for financial support.

${ }^{1}$ S. De Franceschi, L. Kouwenhoven, C. Schönenberger, and W. Wernsdorfer, Nat. Nanotechnol. 5, 703 (2010).

${ }^{2}$ Th. Schäpers, Superconductor/Semiconductor Junctions, Springer Tracts on Modern Physics Vol. 174 (Springer, 2001).

${ }^{3}$ T. Akazaki, H. Takayanagi, J. Nitta, and T. Enoki, Appl. Phys. Lett. 68, 418 (1996).

${ }^{4}$ H. Takayanagi, T. Akazaki, and J. Nitta, Phys. Rev. Lett. 75, 3533 (1995).

${ }^{5}$ Th. Schäpers, V. A. Guzenko, R. P. Müller, A. A. Golubov, A. Brinkman, G. Crecelius, A. Kaluza, and H. Lüth, Phys. Rev. B 67, 014522 (2003).

${ }^{6}$ Y.-J. Doh, J. A. van Dam, A. L. Roest, E. P. A. M. Bakkers, L. P. Kouwenhoven, and S. D. Franceschi, Science 309, 272 (2005).

${ }^{7}$ J. Xiang, A. Vidan, M. Tinkham, R. M. Westervelt, and C. M. Lieber, Nat. Nanotechnol. 1, 208 (2006).

${ }^{8}$ J. A. van Dam, Y. V. Nazarov, E. P. A. M. Bakkers, S. De Franceschi, and L. P. Kouwenhoven, Nature 442, 667 (2006).

${ }^{9}$ R. Frielinghaus, I. E. Batov, M. Weides, H. Kohlstedt, R. Calarco, and Th. Schäpers, Appl. Phys. Lett. 96, 132504 (2010).

${ }^{10}$ S. Roddaro, A. Pescaglini, D. Ercolani, L. Sorba, F. Giazotto, and F. Beltram, Nano Res. 4, 259 (2011).

${ }^{11}$ T. Nishio, T. Kozakai, S. Amaha, M. Larsson, H. A. Nilsson, H. Q. Xu, G. Zhang, K. Tateno, H. Takayanagi, and K. Ishibashi, Nanotechnology 22, 445701 (2011).

${ }^{12}$ V. Mourik, K. Zuo, S. M. Frolov, S. R. Plissard, E. P. A. M. Bakkers, and L. P. Kouwenhoven, Science 336, 1003 (2012).

${ }^{13}$ M. T. Deng, C. L. Yu, G. Y. Huang, M. Larsson, P. Caroff, and H. Q. Xu, e-print arXiv:1204.4130.

${ }^{14}$ A. Das, Y. Ronen, Y. Most, Y. Oreg, M. Heiblum, and H. Shtrikman, e-print arXiv:1205.7073v1.
${ }^{15}$ K. Smit, L. Koenders, and W. Mönch, J. Vac. Sci. Technol. B 7, 888 (1989).

${ }^{16}$ T. Sand-Jespersen, J. Paaske, B. M. Andersen, K. Grove-Rasmussen, H. I. Jørgensen, M. Aagesen, C. B. Sørensen, P. E. Lindelof, K. Flensberg, and J. Nygård, Phys. Rev. Lett. 99, 126603 (2007).

${ }^{17}$ L. Hofstetter, S. Csonka, J. Nygard, and C. Schönenberger, Nature 461, 960 (2009).

${ }^{18}$ P. Spathis, S. Biswas, S. Roddaro, L. Sorba, F. Giazotto, and F. Beltram, Nanotechnology 22, 105201 (2011).

${ }^{19}$ J. C. Cuevas and F. S. Bergeret, Phys. Rev. Lett. 99, 217002 (2007).

${ }^{20}$ F. S. Bergeret and J. C. Cuevas, J. Low Temp. Phys. 153, 304 (2008).

${ }^{21}$ L. Angers, F. Chiodi, G. Montambaux, M. Ferrier, S. Guéron, H. Bouchiat, and J. C. Cuevas, Phys. Rev. B 77, 165408 (2008).

${ }^{22}$ Y.-J. Doh, S. D. Franceschi, E. P. A. M. Bakkers, and L. P. Kouwenhoven, Nano Lett. 8, 4098 (2008).

${ }^{23}$ T. S. Jespersen, M. L. Polianski, C. B. Sørensen, K. Flensberg, and J. Nygård, New J. Phys. 11, 113025 (2009).

${ }^{24}$ H. Takayanagi, J. B. Hansen, and J. Nitta, Phys. Rev. Lett. 74, 166 (1995).

${ }^{25}$ S. Estévez Hernández, M. Akabori, K. Sladek, Ch. Volk, S. Alagha, H. Hardtdegen, M. G. Pala, N. Demarina, D. Grützmacher, and Th. Schäpers, Phys. Rev. B 82, 235303 (2010).

${ }^{26}$ S. Wirths, K. Weis, A. Winden, K. Sladek, C. Volk, S. Alagha, T. E. Weirich, M. von der Ahe, H. Hardtdegen, H. Lüth, N. Demarina, D. Grützmacher, and Th. Schäpers, J. Appl. Phys. 110, 053709 (2011).

${ }^{27}$ P. Jarillo-Herrero, J. van Dam, and L. Kouwenhoven, Nature 439, 953 (2006).

${ }^{28}$ E. Pallecchi, M. Gaaß, D. A. Ryndyk, and C. Strunk, Appl. Phys. Lett. 93, 072501 (2008).

${ }^{29}$ H. Courtois, M. Meschke, J. T. Peltonen, and J. P. Pekola, Phys. Rev. Lett. 101, 067002 (2008).

${ }^{30}$ M. Octavio, M. Tinkham, G. E. Blonder, and T. M. Klapwijk, Phys. Rev. B 27, 6739 (1983).

${ }^{31}$ K. Flensberg, J. B. Hansen, and M. Octavio, Phys. Rev. B 38, 8707 (1988).

${ }^{32}$ J. C. Cuevas, J. Hammer, J. Kopu, J. K. Viljas, and M. Eschrig, Phys. Rev. B 73, 184505 (2006).

${ }^{33}$ G. E. Blonder, M. Tinkham, and T. M. Klapwijk, Phys. Rev. B 25, 4515 (1982).

${ }^{34}$ I. E. Batov, Th. Schäpers, A. A. Golubov, and A. V. Ustinov, J. Appl. Phys. 96, 3366 (2004)

${ }^{35}$ A. Chrestin, T. Matsuyama, and U. Merkt, Phys. Rev. B 55, 8457 (1997).

${ }^{36}$ J. P. Carbotte, Rev. Mod. Phys. 62, 1027 (1990).

${ }^{37}$ J. C. Hammer, J. C. Cuevas, F. S. Bergeret, and W. Belzig, Phys. Rev. B 76, 064514 (2007).

${ }^{38}$ A. M. S. Macêdo and J. T. Chalker, Phys. Rev. B 49, 4695 (1994).

${ }^{39}$ C. W. J. Beenakker, Phys. Rev. Lett. 67, 3836 (1991).

${ }^{40}$ C. W. J. Beenakker and B. Rajaei, Phys. Rev. B 49, 7499 (1994).

${ }^{41}$ B. Al'tshuler and B. Spivak, Zh. Eksp. Teor. Fiz. 92, 609 (1987) [Sov. Phys. JETP 65, 343-347 (1987)].

${ }^{42}$ M. Houzet and M. A. Skvortsov, Phys. Rev. B 77, 024525 (2008).

${ }^{43}$ Th. Schäpers, A. Kaluza, K. Neurohr, J. Malindretos, G. Crecelius, A. van der Hart, H. Hardtdegen, and H. Lüth, Appl. Phys. Lett. 71, 3575 (1997).

${ }^{44}$ A. D. Stone, Phys. Rev. Lett. 54, 2692 (1985).

${ }^{45}$ B. Al'tshuler, Pis'ma Zh. Eksp. Teor. Fiz. 41, 530 (1985) [JETP Lett. 41, 648-651 (1985)].

${ }^{46} \mathrm{Ch}$. Blömers, M. I. Lepsa, M. Luysberg, D. Grützmacher, H. Lüth, and Th. Schäpers, Nano Lett. 11, 3550 (2011).

${ }^{47}$ J. Trbovic, N. Minder, F. Freitag, and C. Schönenberger, Nanotechnology 21, 274005 (2010).

${ }^{48}$ C. Ojeda-Aristizabal, M. Ferrier, S. Guéron, and H. Bouchiat, Phys. Rev. B 79, 165436 (2009).

${ }^{49}$ R. M. Lutchyn, J. D. Sau, and S. Das Sarma, Phys. Rev. Lett. 105, 077001 (2010).

${ }^{50}$ Y. Oreg, G. Refael, and F. von Oppen, Phys. Rev. Lett. 105, 077002 (2010).

${ }^{51}$ C. Fasth, A. Fuhrer, L. Samuelson, V. N. Golovach, and D. Loss, Phys. Rev. Lett. 98, 266801 (2007). 